\title{
PROCESSOS, MÉTODOS E PRÁTICAS DE ENGENHARIA DE SOFTWARE EM PROJETOS SOFTWARE LIVRE: UM ESTUDO DE CASO OWNCLOUD E NEXTCLOUD
}

\author{
Processes, methods and practices of Software Engineering in open \\ source projects: A case study of OwnCloud and Nextcloud
}

\author{
Juliano Rodrigues Ramos ${ }^{1}$; Tamiris Fernanda Malacrida ${ }^{2}$ \\ ${ }^{1}$ Universidade do Oeste Paulista - Unoeste \\ Graduado em Sistemas de Informação pela Faculdade de Informática de Presidente \\ Prudente - FIPP \\ Presidente Prudente - São Paulo, Brasil. \\ juliano_rr@yahoo.com.br \\ ${ }^{2}$ Universidade do Oeste Paulista - Unoeste \\ Graduada em Ciência da Computação pela Faculdade de Informática de Presidente \\ Prudente - FIPP \\ Presidente Prudente - São Paulo, Brasil. \\ tamirismalacrida@gmail.com
}

RESUMO - As práticas da Engenharia de Software (ES) têm por fundamento tentar melhorar a qualidade dos softwares, nos quais se incluem os Open Source Software (OSS). O objetivo deste trabalho é identificar as práticas da ES implementadas nos projetos OSS OwnCloud e Nextcloud. Para tanto, realizouse uma revisão da literatura científica, a partir de diferentes bases de dados digitais, bem como foi considerada a literatura cinza. Foi concluído que os projetos de armazenamento em nuvem OwnCloud e Nextcloud adotam boas práticas de engenharia de software. Porém, foi constatado que, o maior foco é ainda na codificação, e que, portanto, os demais processos do desenvolvimento, como as documentações, controle efetivo do gerenciamento das mudanças e processos de gestão da qualidade, devem receber mais ênfase.

Palavras-chave: Software Open Source; OwnCloud; Nextcloud; Engenharia de Software.

ABSTRACT - Software Engineering practices aim to improve the softwares quality, which includes Open Source Software (OSS). This work aims to identify software engineering practices in the OSS projects OwnCloud e Nextcloud. We perform a scientific literature review, using digital databases such as the Google Scholar and CAPES Periodic Portal, and also search using the gray literature. We conclude that good practices of software engineering are applied into both cloud storage projects OwnCloud and Nextcloud. However, we found that the major focus is still on coding, and therefore, other development processes, such as documentation, effective control of change

Recebido em: 02/06/2018 Revisado em: 04/09/2018 Aprovado em: 17/09/2018 management and quality management processes, should receive more emphasis.

Keywords: Open Source Software; OwnCloud; Nextcloud; Software Engineering. 


\section{INTRODUÇÃO}

O desenvolvimento de software tem crescido nos últimos anos devido a sua enorme necessidade na sociedade contemporânea, e um aspecto de destaque nesse contexto é o volume de Open Source Software (OSS). A Engenharia de Software (ES) surge no sentido de melhorar a qualidade dos softwares em geral e aumentar a produtividade no desenvolvimento de tais produtos (ALGARRÃO, 2018).

Diversas plataformas OSS, para computação em nuvem, foram lançadas nos últimos tempos e dentre algumas das mais populares, tem-se: Eucalyptus, OpenStack, Nimbus, OpenNebula, OwnCloud e Nextcloud (THOMÉ; HENTGES; GRIEBLER, 2013). A computação em nuvem é a convergência e a evolução de vários conceitos da virtualização, projeto de aplicativos distribuídos, da grade e do gerenciamento de Tecnologia da Informação (TI) corporativo, para permitir uma abordagem mais flexível à implantação e ao dimensionamento de aplicativos (THOMÉ; HENTGES; GRIEBLER, 2013).

Em 2010, Frank Karlitschek propõe a primeira versão do OwnCloud, sendo sua versão beta disponibilizada em março do mesmo ano (FELDMAN, 2018). Dois anos depois, surge o OwnCloud Inc, encerrado em abril de 2016, quando Frank Karlitschek propõe o Nextcloud (FELDMAN, 2018). Assim como o OwnCloud, o Nextcloud é um serviço OSS de armazenamento e sincronização de arquivos privados, similar ao Dropbox (proprietário), porém derivado (fork) do OwnCloud. O objetivo do Nextcloud é trazer diversas funcionalidades e melhorias ao projeto inicial, o OwnCloud, o qual sofreu algumas divergências na comunidade para se manter no mercado (OwnCloud e Nextcloud: Comparando Serviços de Armazenamento em Nuvem).

A partir do desafio fundamental da Engenharia de Software que se resume a 'como construir softwares melhores?', este trabalho tem por objetivo identificar as práticas da ES implementadas nos projetos OSS OwnCloud e Nextcloud.

\section{MÉTODO}

O método empregado para atingir o objetivo proposto deste trabalho consistiu da revisão da literatura, a partir de diferentes bases de dados digitais como Google Scholar e Portal de Periódicos CAPES. Além disso, considerou-se a denominada literatura cinza, a qual inclui a pesquisa em blogs e sites, isto devido ao fato tanto da dificuldade de se encontrar trabalhos científicos relacionados ao tema em estudo, quanto por observar ser recorrente o uso da literatura cinza no desenvolvimento de trabalhos científicos na área da computação como, por exemplo, realizado por Afzal et al. (2016) e Garousi, Ferderer e Hacaloglu (2017; 2018).

\section{RESULTADOS E DISCUSSÃO}

Os resultados e as respectivas discussões consistem das práticas da engenharia de software aplicadas nos projetos OwnCloud e Nextcloud, e são apresentados nas subseções que seguem.

\subsection{Gerenciamento de Projetos}

O planejamento de projeto é a documentação que reúne e organiza todos os processos da fase de planejamento e, normalmente, é elaborado por uma equipe que trabalha somente para definições de projetos. Nesse planejamento, define-se como o projeto será executado, monitorado, controlado e encerrado, planejando todas as ações necessárias para que os objetivos propostos sejam alcançados (ALGARRÃO, 2018).

Em se tratando do fork do OwnCloud para o Nextcloud, não houve um planejamento de projeto adequado de como seria o processo de migração, se comparado aos itens da Engenharia de Software. Isto porque foi uma surpresa para todos os usuários quando o fundador da OwnCloud, Frank Karlitschek, uma comunidade com mais 
de 10 milhões de membros, anuncia sua saída para formar uma nova empresa (OwnCloud e Nextcloud: Comparando Serviços de Armazenamento em Nuvem). Com isso, o OwnCloud teve como planejamento continuar fornecendo software e suporte, com sua equipe original, para não sofrer decadência e correr o risco de ir à falência (BHARTIYA, 2016). Nesse contexto, o Nextcloud ganhou forças para se construir devido grande parte de sua equipe ser oriunda do OwnCloud, a qual possuía vasta experiência na área para a construção de um novo projeto (BHARTIYA, 2016).

\subsection{Engenharia de Requisitos}

A gestão de requisitos em projetos de código aberto, geralmente, é complexa de se fazer uma vez que os requisitos de usuários estão espalhados em um volume extenso e em uma variedade de fontes, como fóruns de discussão, blogs e etc, além de apresentarem uma alta porcentagem de duplicações em vários projetos de alto nível (HECK; ZAIDMAN, 2013).

Boa parte dos projetos de software, OSS ou projetos industriais, utilizam algum tipo de metodologia de desenvolvimento ágil de software, onde a forma mais comum de representação dos requisitos é no formato de User Stories (US). Apesar de sua popularidade na indústria de software, quase não se encontram pesquisas de avaliação quanto à sua qualidade. Os post-its utilizados são, muitas vezes, fonte de textos informais que podem conter duplicidades ou informações incoerentes (LUCASSEN et al., 2015).

Quanto aos projetos OwnCloud e Nextcloud, estes permitem o apontamento de bugs e novas funcionalidades em formato de issues na própria ferramenta que gerencia o repositório de cada um dos seus projetos, o GitHub (OwnCloud Core - Issues) e (Nextcloud Core - Issues). Além disso, tais projetos possuem um portal central de atendimento para a recepção de situações apontados pelos usuários que desejam reportar problemas ou colaborar com os projetos de alguma forma. Esses portais de inclusão de US permitem a localização das histórias por categoria e popularidade, o que auxilia na localização de uma situação já existente, evitando duplicidades (Central de suporte OwnCloud) e (Central de suporte Nextcloud).

\subsection{Gerência de configuração de software}

A Gerência de Configuração de Software (GCS) se resume no conjunto de atividades que permite a absorção ordenada das mudanças inerentes ao desenvolvimento de software: controlar e acompanhar mudanças (Controle de Mudança), registrar a evolução do projeto (Controle de Versão) e estabelecer a integridade do sistema (Integração Contínua) (DIAS, 2016).

O controle de mudanças na maioria dos projetos de software livre, utiliza a própria aplicação onde está o repositório para o reporte de bugs e solicitação de mudanças. Isto não é muito diferente para as aplicações OwnCloud e Nextcloud. Ambos, a partir do seu MD (Manual do Desenvolvedor), oferecem acesso ao nomeado Bugtracker, um portal onde é possível encontrar as diretrizes para o envio de problemas (Nextcloud Developer's Manual) e (Support Channels - OwnCloud). Trata-se de um FAQ (Frequently Asked Questions), no qual os reports podem ser classificados com as categorias label e tags. Além disso, bugs e novas funcionalidades, também, são relatadas em formato de issues no GitHub, no qual pedem que sejam realizadas no repositório correspondente, o repositório do projeto cliente ou servidor.

Quanto ao controle de versão, esta é a parte principal da GCS, pois gerencia o registro histórico de cada incremento (configuração) de implementação de solicitação de mudança. Além do registro das configurações, o controle de versão possibilita a edição concorrente sobre os arquivos e a criação de variações dos projetos (FELDMAN, 2018).

Em projetos OSS, a implementação de código, em sua maioria em formato de requisições de pacotes de código (pull- 
requests), é a parte priorizada pelas comunidades open source (STEINMACHER et al., 2018). As submissões voluntárias de correção de erros e propostas de novos recursos são de extrema importância para a continuidade dos projetos do tipo OSS.

Os projetos OwnCloud e Nextcloud possuem seu controle de versão de código em repositórios do GitHub. O passo a passo das submissões está bem documentado nos manuais do desenvolvedor de cada uma dessas aplicações (CHEN; JIANG, 2017) e (Developer's Manual OwnCloud). Ambos os manuais são bem semelhantes e bem completos, contendo informações desde o download do código fonte, requisitos de plugins e aplicações a serem instaladas, até orientações sobre como deve ser feita a submissão da contribuição, por exemplo, referência quanto à estrutura do código e obrigatoriedade de testes. Além disso, está bem clara a orientação para que sejam, primeiramente, consultadas as listas de issues existentes em cada respectivo repositório para evitar submissões não aceitas como em (STEINMACHER et al., 2018).

Em relação à Integração Contínua $(\mathrm{Cl})$, esta consiste em construir o sistema a partir dos itens registrados em uma configuração, e em sua maioria é feita através de scripts que automatizam a construção, testes e coleta de métricas de qualidade (FELDMAN, 2018). As ferramentas de integração contínua acompanham o controle de versão e disparam os scripts cada vez que uma nova configuração é registrada (FELDMAN, 2018).

Inicialmente, a OwnCloud utilizou as ferramentas de integração contínua OSS Travis e Jenkins. No entanto, utilizando apenas a versão gratuita do Travis, e definindo não ser possível o investimento pelo alto custo da versão premium, a empresa se deparou, ainda, com problemas de complexidade, utilização na produção e demora na execução dos testes (JACKSON, 2018). Na utilização do Jenkins, a equipe do OwnCloud teve muitos problemas relacionados a atualização, espaço em disco, gerenciamento dos containers, além de problemas de lentidão (JACKSON, 2018).

Com o intuito de minimizar tantos impeditivos de $\mathrm{Cl}$, a equipe de engenheiros e arquitetos da OwnCloud optou por adotar a ferramenta Drone, também um sistema de $\mathrm{Cl}$ de código aberto com empacotamento em containers. Além de eliminar os problemas de $\mathrm{Cl}$ existentes, esta mudança permitiu que a empresa aumentasse a quantidade de testes de unidades e integrações feitas em recursos novos e ou atualizados (JACKSON, 2018). Conforme relatado por um dos desenvolvedores da Nextcloud (WURST, 2018), a empresa utiliza atualmente como práticas de $\mathrm{Cl}$ as ferramentas open source Xmllint e Travis.

\subsection{Qualidade de Software}

A premissa básica de vários estudos sobre a qualidade de software é que a qualidade do produto depende da qualidade do processo de desenvolvimento (KOSCIANSKI; SOARES, 2007). Neste sentido, a busca por um software de qualidade passa naturalmente pela necessidade de melhoria no processo de desenvolvimento.

Existem diversas evidências em projetos open source sobre a utilização de boas práticas de melhoria de qualidade nas aplicações produzidas, conforme relatado, por exemplo, em (Developer's Manual Owcloud), (Developer's Manual Nextcloud).

Sendo o foco no controle da codificação, as duas etapas conhecidas para consideração da qualidade nos projetos OSS são revisão do código-fonte e testes automatizados (STEINMACHER et al., 2018) e (CHEN; JIANG, 2017). A primeira é realizada, em grande parte dos projetos, por um ou mais moderadores, isto é, integrador(es) que revisa $(m)$ as requisições de pacotes de código (pull-requests) antes de integrá-los ao branch principal e torná-los parte do produto (CHEN; JIANG, 2017). A etapa de testes automatizados trata da codificação de testes que são executados de forma automática e que devem garantir a cobertura dos módulos principais executados sem erro 
(STEINMACHER et al., 2018). Projetos OSS grandes e mais controlados utilizam como critério não somente a validação da qualidade do código apresentado (existência de anti-padrões) (CHEN; JIANG, 2017), mas também a existência da codificação dos testes para aceitar ou não uma submissão de alteração (STEINMACHER et al., 2018). As comunidades OwnCloud e Nextcloud não são diferentes nestes aspectos. Os manuais de desenvolvedores de ambas as empresas exprimem objetivamente e da mesma forma que qualquer submissão de pull-request, a submissão não é aceita antes de ser revisada no mínimo por dois colaboradores ainda que seja referente a uma única linha (Developer's Manual Nextcloud).

De acordo com (CHEN; JIANG, 2017) e (Developer's Manual Owcloud), as comunidades OwnCloud e Nextcloud não aceitam pedidos de pull-request enviados com código não testado. Testes de unidade são realizados com o framework PHPUnit e testes de interface utilizando Selenium com apoio da aplicação Source Labs. Referente às documentações ambas, OwnCloud (https://doc.OwnCloud.com/) e Nextcloud (https://docs.Nextcloud.com/), possuem um wiki específico para as documentações, onde mantém manuais de usuários, administração e desenvolvedor para cada uma das versões disponíveis, além de manterem um manual de orientação de contribuições.

\subsection{Gerenciamento de aquisições}

Grande parte dos produtos OSS licenciados utilizam de um tipo de licenciamento específico para projetos de código aberto, como a GPL. Em vez de comprar o produto, você compra suporte e serviços, garantindo qualidade e continuidade (Why the (A) GPL is great for business users).

Desenvolvidas com informações de advogados de todo o mundo, as licenças de software GPL e sua derivada para software Web, AGPL, são as licenças copyleft mais usadas, aceitas, comprovadas e testadas pela comunidade de software, e projetadas principalmente para proteger o destinatário do código contra abuso por parte do fornecedor (Why the (A) GPL is great for business users).

Tanto o OwnCloud quanto o Nextcloud são licenciados sobre a $A G P L$ v3. Sendo que, em junho de 2017, a Nextcloud tomou a iniciativa de ser a primeira solução de sincronização e compartilhamento de arquivos corporativos para verificar a conformidade total da licença por meio do OpenChain, um projeto importante da Linux Foundation que identifica os principais processos recomendados para o gerenciamento eficaz de código aberto (Plans and Pricing for Nextcloud Files).

\section{CONCLUSÃO}

Este trabalho realizou a identificação das práticas da Engenharia de Software aplicadas em projetos de software livre de armazenamento em nuvem, o OwnCloud e o Nextcloud. Foi constatado que, existem diversas pesquisas referentes às práticas de Engenharia de Software em aplicações open source. Todavia, em sua maioria, o maior foco é na codificação, não se atentando aos demais processos do desenvolvimento, como as documentações, controle efetivo do gerenciamento das mudanças e processos de gestão da qualidade. Embora boa parte dos processos de desenvolvimento não sejam abertos ao público, acredita-se que muitos processos estão incorporados e inclusive procedimentos de melhoria de processos são realizados no dia a dia de algumas das comunidades OSS, principalmente em projetos grandes como OwnCloud e Nextcloud que possuem parte dos seus produtos ou serviços comercializados e uma vasta equipe de colaboradores efetivos.

Foi concluído que, no projeto Nextcloud, por se tratar de um projeto fork recente, a maioria das características documentadas dos processos de fábrica do software ainda estão semelhantes ou exatamente iguais ao OwnCloud. Porém, verificou-se uma evolução na integração 
contínua com a adoção da aplicação Drone por parte da OwnCloud, o que mostra a tentativa pela busca por algum diferencial competitivo.

Em trabalhos futuros, foi sugerido um estudo que compare outras aplicações de armazenamento de dados em nuvem, elencando as diferenças entre as aplicações estudadas.

\section{REFERÊNCIAS}

AFZAL, W. et al. Software test process improvement approaches: a systematic literature review and an industrial case study. Journal of Systems and Software, v. 111, p. 1-33, 2016. https://doi.org/10.1016/j.jss.2015.08.048

ALGARRÃO, P. A. Processos e fases de gerenciamento de projetos. Universidade de Cândido Mendes, 2018.

BHARTIYA, S. OwnCloud bifurcou-se para criar o Nextcloud. 2016. Disponível em: https://www.cio.com/article/3078173/cloudcomputing/OwnCloud-forked-to-createNextcloud.html. Acesso em: jul. 2018.

CHEN, B.; JIANG, Z. M. Characterizing and detecting anti-patterns in the logging code. In: INTERNATIONAL CONFERENCE ON SOFTWARE ENGINEERING, 39. Proceedings... Argentina, 2017.

DIAS, A. F. O que é Gerência de Configuração de Software?. maio, 2016. Disponível em: https://blog.pronus.io/posts/o-que-ehgerencia-de-configuracao-de-software/. Acesso em: mar. 2018.

FELDMAN, D. A história do OwnCloud e Nextcloud. Disponível em: https://civihosting.com/blog/Nextcloud-vsOwnCloud/. Acesso em: jul. 2018.

GAROUSI, V; FELDERER, M.; HACALOĞLU, T. What we know about software test maturity and test process improvement. IEEE
Software, v. 35, n. 1, p. 84-92, 2018. https://doi.org/10.1109/MS.2017.4541043

GAROUSI, V.; FELDERER, M.; HACALOĞLU, T. Software test maturity assessment and test process improvement: a multivocal literature review. Information and Software Technology, v. 85, p. 16-42, 2017. https://doi.org/10.1016/i.infsof.2017.01.001

HECK, P.; ZAIDMAN, A. An analysis of requirements evolution in open source projects: recommendations for issue trackers. In: INTERNATIONAL WORKSHOP ON PRINCIPLES OF SOFTWARE EVOLUTION, August 19-20, 2013. Proceedings... Saint Petersburg, Russia. 2013.

JACKSON, J. How Drone Solved OwnCloud's Continuous Integration Woes. Disponível em: https://thenewstack.io/how-dronesolved-OwnClouds-continuous-integrationwoes/. Acesso em: jul. 2018.

KOSCIANSKI, A.; SOARES, M. S. Qualidade de Software. 2. ed. São Paulo: Novatec, 2007.

LUCASSEN, G. et al. Forging high-quality user stories: towards a discipline for agile requirements. In: IEEE. Proceedings... 2015.

STEINMACHER, l. et al. Almost there: a study on quasi-contributors in open source software projects. In: INTERNATIONAL CONFERENCE ON SOFTWARE ENGINEERING, SWEDEN. Proceedings... 2018.

THOMÉ, B.; HENTGES, E.; GRIEBLER, D. Computação em nuvem: análise comparativa de ferramentas open source para iaas. In: ESCOLA REGIONAL DE REDES DE COMPUTADORES (ERRC),11. Anais... 2013. 
WURST, C. Developer at Nextcloud GmbH, informal guide of Continuous integration best practices. Mar. 2018.

https://blog.wuc.me/2018/03/06/validate-

Nextcloud-info-xml.html. Acesso em: jul. 2018. 